\title{
Kenaf (Hibiscus cannabinus L.) Leaves and Seed as a Potential Source of the Bioactive Compounds: Effects of Various Extraction Solvents on Biological Properties
}

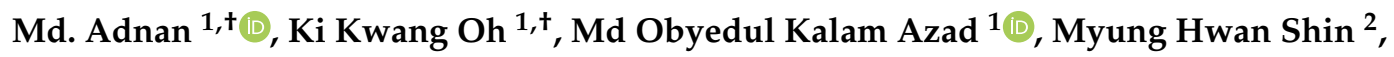 \\ Myeong-Hyeon Wang ${ }^{3, *}$ and Dong Ha Cho ${ }^{1, *}$ \\ 1 Department of Bio-Health Technology, College of Biomedical Sciences, Kangwon National University, \\ Chuncheon 24341, Korea; mdadnan1991.pharma@gmail.com (M.A.); nivirna07@kangwon.ac.kr (K.K.O.); \\ azadokalam@gmail.com (M.O.K.A.) \\ 2 SJ Global Ltd., Gyeongsangnam do, Busan 635-890, Korea; dsd78@naver.com \\ 3 Department of Medical Biotechnology, College of Biomedical Sciences, Kangwon National University, \\ Chuncheon 24341, Korea \\ * Correspondence: mhwang@kangwon.ac.kr (M.-H.W.); chodh@kangwon.ac.kr (D.H.C.); \\ Tel.: +82-332-506-475 (D.H.C.) \\ + These authors contributed equally to this work.
}

Received: 9 September 2020; Accepted: 25 September 2020; Published: 28 September 2020

\begin{abstract}
Hibiscus cannabinus (Kenaf) is a potential source of bioactive constituents and natural antioxidant. The current study determined the impact of various solvents on extraction yield, recovery of polyphenol and flavonoid, antioxidant, anticancer, and antibacterial properties of Kenaf leaves and seed. The powder of leaves and seed was separately extracted with $n$-hexane, ethyl acetate, ethanol, and water solvent. Among them, the ethanol extract of leaves and seed showed the highest extraction yield, and their GC-MS analysis revealed a total of 55 and 14 bioactive compounds, respectively. The total polyphenols (TP) and flavonoids (TF) content were quantified by a spectrophotometric technique where water extracts displayed a noteworthy amount of TP and TF content compared to other extracts. A similar demonstration was noticed in antioxidant activity, evaluated by DPPH (2,2-diphenyl-1-picrylhydrazyl) and hydrogen peroxide scavenging capacity. In addition, cytotoxicity and anti-lung cancer activity were identified against mouse embryonic fibroblast (NIH3T3) and human lung cancer (A549) cells. All extracts of leaves and seed were observed as non-toxic to the NIH3T3 cells, but slight toxicity was expressed by $n$-hexane extracts at the optimum dose $(1000 \mu \mathrm{g} / \mathrm{mL})$ of treatment. In parallel, $n$-hexane and ethanol extracts (leaves and seed) exposed promising anti-lung cancer activity at the same concentration. Furthermore, antibacterial activity was assessed using disc diffusion assay, and seed extracts exhibited a significant inhibition zone against Gram-positive and Gram-negative microorganisms. Overall, Kenaf seed extracted with polar solvents was found very potent in terms of important bioactive compounds and pharmacological aspects, which can be an excellent biological matrix of natural antioxidants.
\end{abstract}

Keywords: Kenaf leaves and seed; solvent extractions; GC-MS; antioxidant; cytotoxicity and anti-lung cancer; and antibacterial

\section{Introduction}

One of the common manifestations of living cells is the generation of harmful pro-oxidants and reactive oxygen species (ROS), produced either due to the biological dysfunctions or as a result of cellular metabolisms (byproducts) [1]. Such free radicals may induce oxidative damage to proteins, lipids, and nucleic acids and lead to several life-threatening conditions, including cancer, neurodegenerative 
disease, ischemic heart disease, diabetes mellitus, and other chronic diseases [2,3]. In regards to preventing oxidative stress, antioxidants with adequate scavenging capacity have been used for appropriate balancing through regulation of oxidation or auto-oxidation processes. Commonly, many synthetic antioxidants, including butylated hydroxytoluene (BHT), tertiary butyl hydroquinone (TBHQ), and butylated hydroxyanisole (BHA) are widely added during food processing, preservation, as well as when preventing deterioration of color and texture [4]. However, these antioxidants have emerged as hazardous for human health in recent times [5]. Hence, researchers have refocused on alternative sources of antioxidants, especially from plant-derived products, which are very common in traditional medicine. Since plants contain heterogeneous metabolites and biomolecules in their compositions, with potential defense mechanisms, their synergistic action in treating various chronic diseases is an area of intense interest [6,7]. Therefore, investigation of valuable plants that possess abundant phenolics and other bio-active compounds must be screened meticulously in search for novel bioactive and safe antioxidants.

Cancer has 36 different types, and is a major burden for the world. In 2018, 18.1 million of new cases and 9.6 million deaths were recorded, where collateral, liver, lung, thyroid, and stomach cancer were highly prevalent in both men and women [8]. By 2030, it is foretold that the new suspected cases and death toll by cancer will surpass around 26 million and 17 million, respectively [9]. Hence, researchers are searching for an alternative method using novel chemotherapeutic agents, which are most importantly safe, non-toxic, and easily available. In this regard, medicinal plants, traditional medicine, phytomedicine, and the pharmacological potential of plant compounds are the best choice. Currently, $60 \%$ of approved anticancer drugs have been derived from a natural source [10]. Besides, polyphenols and antioxidant-rich fruits and vegetables can also play a pivotal role in malignancy transformation and cancer development [11]. Analogues to cancer, bacterial resistance to all classes of antibiotics are one of the most alarming issues for global public health. In recent decades, no significant antibiotics have been discovered which urge the development of new drugs with novel mechanisms of action against various pathogens [12]. Due to the high chemical diversity, bioactive compounds and metabolites extracted from natural products are considered as viable candidates for bioprospecting programs, which can intervene in a range of microbial pathways [13].

Kenaf (Hibiscus cannabinus) is an annual herbaceous dicotyledonous plant, belongs to the Malvaceae family, is widely distributed in Asia and Africa, and grows mostly in temperate to tropical areas [8]. Kenaf (leaves and seed) has many significant medicinal properties, including anticancer, antioxidants, analgesic, anti-inflammatory, aphrodisiacs, and hepatoprotective activities [9,10]. In traditional medicine, Kenaf is used to treat various diseases; for instance, a paste of the leaf and stem is used to treat Guinea worms disease and anemia in Africa [10]. Moreover, in ayurvedic medicine, the leaves are used to treat various disorders, such as of the blood, diabetes, bilious, the throat, and coughs $[10,11]$. Furthermore, flower juice and seed are consumed for biliousness and bruises [12]. These medicinal benefits are exposed due to the presence of abundant phenylpropanoid compounds in the Kenaf plant [13]. Besides, many bioactive compounds, such as omega-3 fatty acids and sterols [11,14], as well as phenolic compounds, including kaempferol, vanillic acid, syringic acid, caffeic acid, gallic acid, p-hydroxybenzoic acid, p-coumaric, and ferulic acid have been identified earlier from seed extracts [12]. Previously, Kenaf seed was used to prepare biopolymer-mediated nanocomposites to enhance seed flour's antioxidant capacity [5]. In addition, efficient silver nanoparticles were synthesized using the seed extract, which manifested promising antibacterial and anticancer activities [15].

However, despite having such important biological properties of different parts of this plant, a few scientific reports have been found based on its pharmacological aspect. Hence, in this study, we aimed to identify the content of secondary metabolites, antioxidant, anticancer, and antibacterial properties of Kenaf leaves and seed. To evaluate this, various solvents were used to extract effective phytoconstituents in a particular solvent. Besides, ethanol extract of both leaves and seed were explored with the aid of gas chromatography-mass spectrometry (GC-MS) analysis. In particular, this research 
demonstrates the influence of various solvent extracts on the Kenaf leaves and seed, and also reveals their pharmacological activities.

\section{Method}

\subsection{Chemicals}

Ethanol, Ethyl acetate, $n$ hexane, phenolic reagent (Folin-Ciocalteu, $2 \mathrm{~N}$ ), sodium carbonate $\left(\mathrm{Na}_{2} \mathrm{CO}_{3}\right)$, aluminum nitrate $\left(\mathrm{AlNO}_{3}\right)$, potassium acetate $\left(\mathrm{CH}_{3} \mathrm{COOK}\right)$, DPPH (2,2-diphenyl-1 -picrylhydrazyl), hydrogen peroxide $\left(\mathrm{H}_{2} \mathrm{O}_{2}\right)$, Mueller Hinton agar media, and ampicillin were procured from Sigma (Sigma Chemical Co., St. Louis, MO, USA). Other chemicals, such as a water-soluble tetrazolium (WST) assay kit (EZ-Cytox, Daeil Lab Service, Gwangiu, Korea), PBS, Dulbecco's modified eagle medium (DMEM), fetal bovine serum (FBS), Roswell Park Memorial Institute (RPMI) medium, and penicillin-streptomycin (PS) were purchased from Gibco (Waltham, MA, USA) and Thermo Fishers Scientific (Seoul, Korea). The NIH3T3 A549 cells were collected from the Korean Cell Line Bank (KCLB, Seoul, Korea).

\subsection{Plant Material and Extracts Preparation}

The leaves (younger completely formed leaves) and seed (collected before harvesting) of Kenaf (Israeli verity) were supplied by the Kenaf Company (Gangwondaehak-gil, Chuncheon-si, Gangwon-do, Korea, 24341). The collected samples (leaves and seed) were cleaned and placed to be oven-dried for a week by maintaining a suitable temperature $\left(55^{\circ} \mathrm{C}\right)$. Afterwards, the dried samples (water content was zero) were pulverized into a coarse powder using a pin crusher (JIC-P10-2; Myungsung Machine, Seoul, Korea). The ground samples were passed through $300 \mu \mathrm{m}$ mesh-size sieves to form a fine powder, and stored at room temperature before extract preparation.

The fine powders of Kenaf leaves (500 g) and seed ( $500 \mathrm{~g}$ ) were soaked separately in $2.5 \mathrm{~L}$ of ethanol for five days at room temperature, with continuous stirring and shaking on a Rotary Shaker (JEIOTECH SI-900 R). Afterwards, the solvent extracts were first filtered by a sterilized cotton plug, and then we used Whatman filter paper No. 1, followed by evaporation through the rotary evaporator at $50{ }^{\circ} \mathrm{C}$ to get semisolids of Kenaf leaves ( $36 \mathrm{~g}$ ) and seed extract $(41 \mathrm{~g})$. The same process was repeated for $n$-hexane, ethyl acetate, and water solvent extraction. After evaporation (above mentioned condition) of each solvent, the yield of $n$-hexane (leaves: $3.17 \mathrm{~g}$; seed: $4.27 \mathrm{~g}$ ), ethyl acetate (leaves: $6.17 \mathrm{~g}$; seed: $10.76 \mathrm{~g}$ ), and water (leaves: $8.12 \mathrm{~g}$; seed: $9.29 \mathrm{~g}$ ) extract were collected and preserved in a refrigerator for further investigation. In order to investigate the pharmacological potentials, the samples were prepared in 5\% di-methyl sulfoxide (DMSO).

The yield after extraction was calculated as follows:

Yield $(\%)=($ Dried extract weight $/$ Dried sample weight $) \times 100$.

\subsection{Gas Chromatography-Mass Spectroscopy (GC-MS) Analysis of Kenaf (Leaves and Seed) Extracts}

The bioactive compounds of Kenaf leaves and seed (ethanol extract) were detected by GC-MS analysis using an Agilent Technologies 7890A capillary gas chromatograph, along with a mass spectrometer system. GC was equipped with a $30 \mathrm{~m} \times 0.25 \mathrm{~mm} \times 0.25 \mu \mathrm{m}$ DB- 5 capillary column. Initially, the instrument was maintained at a temperature of $100^{\circ} \mathrm{C}$ for $2 \mathrm{~min}$ and $6 \mathrm{~s}$. The temperature was risen to $300{ }^{\circ} \mathrm{C}$ at the rate of $25{ }^{\circ} \mathrm{C} / \mathrm{min}$ and maintained for $20 \mathrm{~min}$ at the end of this period. The injection port temperature and the helium flow rate were ensured to be $250{ }^{\circ} \mathrm{C}$ and $1.5 \mathrm{~mL} / \mathrm{min}$, respectively. The ionization voltage was $70 \mathrm{eV}$. The sample was injected in split mode at 5:1. The MS scan range was set at 35-550 ( $\mathrm{m} / \mathrm{z})$. The fragmentation patterns of mass spectra were compared with those stored in the using W8N05ST Library MS database. The percentage of each compound was calculated from the relative peak area of each compound in the chromatogram. The concept of integration used the Chem Station integrated algorithms. 


\subsection{Quantitative Analysis}

\subsubsection{Total Phenolic Content (TPC)}

The total content of phenol in Kenaf leaves and seed extracts was assessed following the method of Folin-Ciocalteau [16]. The Folin-Ciocalteau reagent $(200 \mu \mathrm{L}, 1 \mathrm{~N})$ was added in the test tube containing $1 \mathrm{~mL}$ of the sample $(10 \mathrm{mg} / \mathrm{mL})$. The volume of the mixture was increased by the addition of deionized water $(1.8 \mathrm{~mL})$ and kept ( $3 \mathrm{~min}$ at room temperature) for the reaction after the vortex. Afterwards, $400 \mu \mathrm{L}$ of sodium carbonate $(10 \% v / v)$ was added to the reaction mixture. Finally, the volume was adjusted up to $4 \mathrm{~mL}$ by adding deionized water $(600 \mu \mathrm{L})$. The mixture was placed in dark ambience for incubation ( $1 \mathrm{~h}$ at room temperature), and the test was done in triplicates. The absorbance was measured against the blank (water) at $725 \mathrm{~nm}$ by the spectrophotometer (UV-1800 $240 \mathrm{~V}$, Shimadzu corporation, Kyoto, Japan). The TPC was calculated from a calibration curve (plotting the value of absorbance vs. concentration) using gallic acid and expressed as mg of GAE (gallic acid equivalent) per $100 \mathrm{~g}$ of extract.

\subsubsection{Total Flavonoid Content (TFC)}

The total flavonoid content in Kenaf leaves and seed extracts was determined according to the previously described method [17] with some modifications [18]. In brief, $0.5 \mathrm{~mL}$ aliquot of extract $(10 \mathrm{mg} / \mathrm{mL})$ was mixed with $100 \mu \mathrm{L}$ of aluminum nitrate $(10 \% w / v), 100 \mu \mathrm{L}$ of potassium acetate $(1 \mathrm{M})$, and $3.3 \mathrm{~mL}$ of ethanol. The mixture was vortexed and placed for incubation (40 $\mathrm{min}$ at room temperature). TF content of the extracts was measured at $415 \mathrm{~nm}$ by the spectrophotometer (UV-1800 $240 \mathrm{~V}$, Shimadzu corporation, Kyoto, Japan) and expressed in mg/100 g QE (quercetin equivalent).

\subsection{Antioxidant Activity}

\section{DPPH Free Radical and Hydrogen Peroxide $\left(\mathrm{H}_{2} \mathrm{O}_{2}\right)$ Scavenging Activity}

The antioxidant activity of Kenaf leaves and seed extracts was evaluated using DPPH free radical and $\mathrm{H}_{2} \mathrm{O}_{2}$, following the method of Braca et al. [19] and Adnan et al. (2020) [5]. For the DPPH, $3 \mathrm{~mL}$ of freshly prepared DPPH $(0.004 \% w / v$ in methanol) was added to the $0.5 \mathrm{~mL}$ of stock solution. The reaction mixture was vortexed and placed in the dark ambience for incubation (30 min at room temperature). For $\mathrm{H}_{2} \mathrm{O}_{2}$ scavenging, $0.6 \mathrm{~mL}$ of $\mathrm{H}_{2} \mathrm{O}_{2}$ solution ( $4 \mathrm{mM}$ prepared with $0.1 \mathrm{M}$ phosphate buffer $\mathrm{pH}$ 7.4) was mixed with $0.4 \mathrm{~mL}$ of stock solution and then incubated for $10 \mathrm{~min}$. The scavenging of DPPH and $\mathrm{H}_{2} \mathrm{O}_{2}$ were measured at $517 \mathrm{~nm}$ and $230 \mathrm{~nm}$, respectively by the spectrophotometer (UV-1800 240 V, Shimadzu Corporation, Kyoto, Japan). The percentage of scavenging capacity was calculated against negative control (methanol $+\mathrm{DPPH}$ ) and (stock solution without $\mathrm{H}_{2} \mathrm{O}_{2}$ ) expressed by the following equation: Scavenging effect $(\%)=\left[\left(\mathrm{Abs}_{\mathrm{c}}-\mathrm{Abs}_{\mathrm{s}}\right) / \mathrm{Abs}_{\mathrm{C}}\right] \times 100$, where $\mathrm{Abs}_{\mathrm{c}}$ is the absorbance of control; $\mathrm{Abs}_{\mathrm{s}}$ is the absorbance of DPPH radical $/ \mathrm{H}_{2} \mathrm{O}_{2}+$ sample (extract/standard).

\subsection{Cell Culture, Cytotoxicity, and Anti-Lung Cancer Assay}

The cytotoxicity and anti-lung cancer potentiality of various solvent extracts (leaves and seed) were evaluated against NIH3T3 and A549 cells, employing the WST assay kit. The NIH3T3 and A549 cells were cultured in the penicillin and streptomycin (PS)-incorporated DMEM and RPMI medium, respectively. The cells were incubated $\left(\mathrm{CO}_{2}\right.$ incubator at $37^{\circ} \mathrm{C}$ for $\left.24 \mathrm{~h}\right)$ and the quality of cells (confluences and morphology) were observed under light microscopy. The prepared cells were further used in cytotoxicity and anti-lung cancer analysis. Briefly, the NIH3T3 $\left(5 \times 10^{4}\right)$ and A549 $\left(1 \times 10^{5}\right)$ cells were seeded in the 96-well plates (containing DMEM and RPMI medium, respectively) and incubated $\left(\mathrm{CO}_{2}\right.$ incubator at $37^{\circ} \mathrm{C}$ for $\left.24 \mathrm{~h}\right)$ until they reached the desirable confluence (80-90\%). The incubated cells were treated with the different concentrations $(62.5,125,250,500$, and $1000 \mu \mathrm{g} / \mathrm{mL})$ of extracts and incubated with a similar condition. Finally, Ex-CyTox reagent $(10 \mu \mathrm{L})$ was added to each well and absorbance was recorded at $450 \mathrm{~nm}$. As a negative control, 5\% DMSO was used. From the obtained 
absorbance, the cytotoxicity and cell viability of the extracts were calculated according to the formula described elsewhere [15].

\subsection{Antibacterial Activity}

Antibacterial activity was analyzed using the disc diffusion method [15]. In brief, Mueller-Hinton agar was prepared and placed into Petri dishes for solidification (under laminar airflow). Gram-positive Staphylococcus aureus (ATCC 6538), Bacillus subtilis (ATCC 6633), and Bacillus cereus (ATCC 14579) and Gram-negative Salmonella Typhi (ATCC 29629), Pseudomonas aeruginosa (ATCC 9027), and Escherichia coli (ATCC 8739) microorganisms were cultured overnight. Of each prepared bacteria, $100 \mu \mathrm{L}$ (bacterial inocula were adjusted to $10^{7} \mathrm{CFU} / \mathrm{mL}$ ) was spread smoothly on the agar surface, and then a sterile disc ( $8 \mathrm{~mm}$ diameter) was laid upon an agar plate (seeded). Each extract with desired concentration $\left(50 \mathrm{mg} / \mathrm{mL}\right.$ ) was loaded on these discs and incubated (at $37^{\circ} \mathrm{C}$ for $24 \mathrm{~h}$ ). The zone of inhibition was recorded and measured in $\mathrm{mm}$. The $5 \%$ DMSO was used as a negative control, and as a positive control, ampicillin $(25 \mu \mathrm{g} / \mathrm{mL})$ was used.

\section{Statistical Analysis}

All data were expressed as mean \pm standard deviation (SD) of several measurements. The obtained results (total phenol, flavonoid, and antioxidant activity) were compared among the composition using a paired $t$-test in order to observe the significant differences at the level of $5 \%$. The paired $t$-test between the mean values was analyzed by MINITAB (version 17.0, Minitab Inc., State College, PA, United States). Data of cytotoxicity was analyzed using GraphPad Prism 6.0 statistical software. Values are expressed as mean $\pm \mathrm{SD}(n=3)$ and two-way ANOVA, followed by the Bonferroni test. There was a significant difference when comparing each column to all other columns at ${ }^{*} p<0.05,{ }^{* *} p<0.01$, and ${ }^{* * *} p<0.001$.

\section{Results and Discussions}

\subsection{Extraction Yields}

Various parts of a plant occupy a pool of bioactive compounds containing potential chemical groups which consistently protect both plants and humans from cellular oxidative damage [20]. However, to explore all these major classes of chemicals, efficient extraction techniques, such as maceration, subcritical water extraction, soxhlet extraction, supercritical fluid extraction, and ultrasonic-assisted extraction with suitable solvents are a prerequisite [21]. Importantly, plant-derived phytoconstituents compose numerous chemical characteristics and polarities that are not readily soluble in a particular solvent [22]. Therefore, various solvents with differing polarities must be studied to extract potential bioactive compounds from the plant [23]. Our study used different solvent types to evaluate the impact of extraction yield, and here, the seed extracts exhibited a higher yield than the leaves' extracts (Figure 1). In both cases, the highest and lowest extraction yields were recorded for the ethanol (ETH) and $n$-hexane (NHX) extracts, respectively. After the ethanol extract, the yield differences were observed in another solvent extraction, such as ethyl acetate (EA), followed by the water (WT) extract for the Kenaf seed, and water followed by an ethyl acetate extract for kenaf leaves. Sim et al. [24] reported that pulsed ultrasonic-assisted Kenaf leaves extraction with water showed the highest extraction yield compared to methanol, ethanol, and acetone solvent. Yusri et al. [25] also followed a similar extraction method for Kenaf seed, and the highest extraction yield was obtained for the hexane extract. However, in our study, the conventional extraction method was followed, and the observed variation of yield extraction might have been due to the influence of a heterogeneous solvent used with different polarities, and the results are in line with the previously reported extraction yields of rice bran [26] and many medicinal plants [27]. 


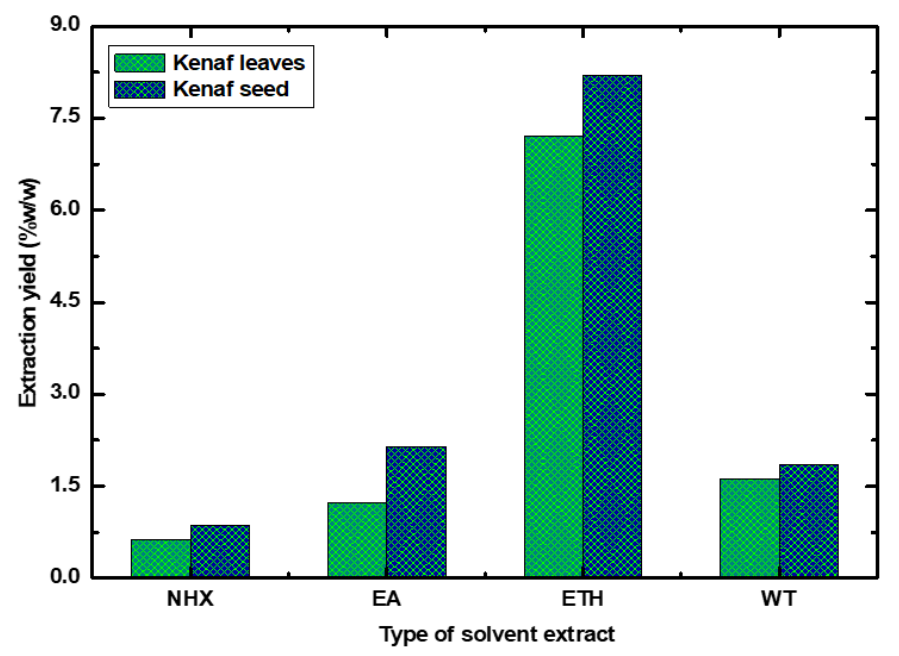

Figure 1. Effect of different solvents on the extraction yield.

\subsection{GC-MS Analysis of Ethanol Extract for Kenaf (Leaves and Seed)}

Since a higher yield was found for the ethanol extract of both Kenaf leaves and seed, we further conducted GC-MS analysis to reveal their chemical profile. It is well-known that GC-MS with specific detection systems is a valuable tool for separating and identifying the components from complex volatile mixtures. GC-MS consists of two analytical techniques where GC splits the component from the mixture, and MS analyzes each of the components separately [28]. In addition, compounds that are small, adequately volatile, and thermostable in the GC environment can be easily analyzed by GC-MS [29]. Previously, GC-MS analysis on the hexane extract of Kenaf leaves and seed manifested 13 and 10 phytoconstituents, respectively [12]. In our study, GC-MS analysis of Kenaf leaves and seed (ethanol extracts) revealed 55 and 14 compounds, having retention times from 3.42 to $18.56 \mathrm{~min}$ and 4.00 to $17.07 \mathrm{~min}$, respectively, which are enlisted in Table 1, and their total ionic chromatograms (TIC) are depicted in Figure 2. Among the 55 compounds in Kenaf leaves, the following components considering their peak area are documented: 5-Hydroxymethylfurfural (7.20\%), 2-Stearoylglycerol (5.44\%), 1,5,9,13-Tetradecatetraene (4.50\%), Vitamin E (4.45\%), alpha-Amyrin (3.76\%), 4H-Pyran-4-one, 2,3-dihydro-3,5-dihydroxy-6-methyl$(3.70 \%)$, Clionasterol (3.93\%), 2-Linoleoylglycerol (3.40\%), and Hexadecanoate (3.06\%). Besides, the detected compounds from Kenaf seed are 9-octadecanoic acid (z)- (77.46\%), Hexadecanoic acid (10.25\%), 9, 12-octadecadienoic acid (z, z)-, 2-hydroxy-1-(hydroxymethyl)ethyl ester (6.21\%), and Linoleic acid $(4.43 \%)$.
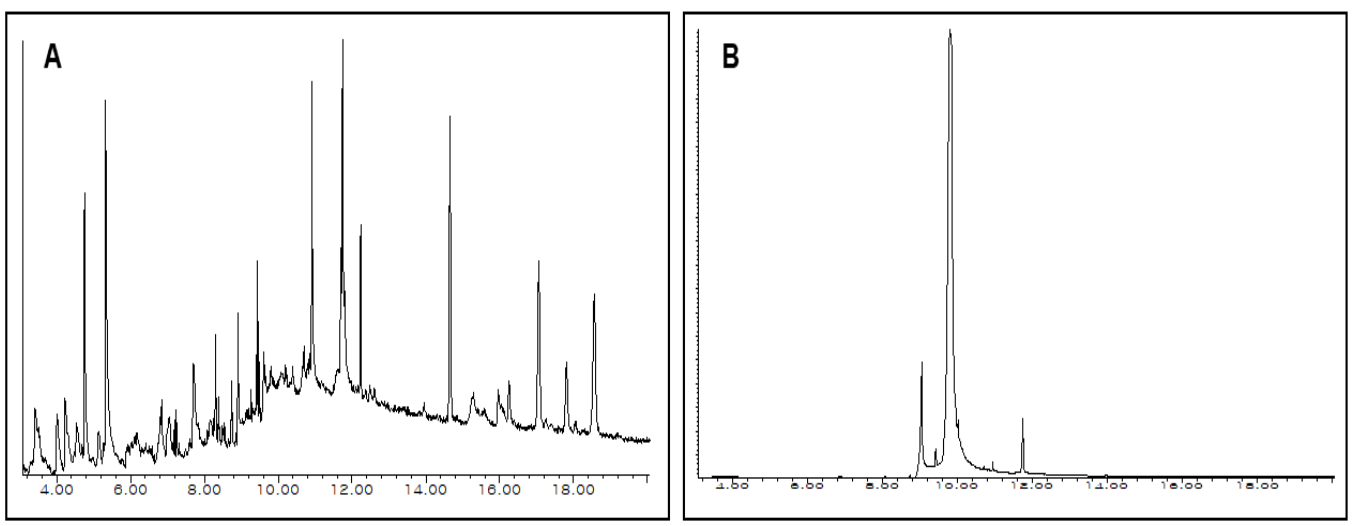

Figure 2. Total ionic chromatogram (TIC) of ethanol extract of Kenaf leaves (A) and seed (B) obtained by GC-MS, with an energy of ionization of $70 \mathrm{eV}$. 
Table 1. List of compounds identified in Kenaf leaves and seed ethanol extract obtained by GC-MS analysis.

\begin{tabular}{|c|c|c|c|c|c|c|}
\hline S/L no & R. Time (min) & PA (\%) & Compound Name & Molecular Formula & MW (g/mol) & *** Activity \\
\hline \multicolumn{7}{|c|}{ Leaves } \\
\hline 1 & 3.42 & 1.56 & 2-(Tert-butylamino)-3-methyl-2-pentenenitrile & $\mathrm{C}_{10} \mathrm{H}_{18} \mathrm{~N}_{2}$ & 166.3 & No activity \\
\hline 2 & 3.51 & 1.25 & 5-Methylfurfural & $\mathrm{C}_{6} \mathrm{H}_{6} \mathrm{O}_{2}$ & 110.1 & $\begin{array}{l}\text { Antioxidant, antiproliferative, } \\
\text { antibacterial }\end{array}$ \\
\hline 3 & 4.01 & 1.75 & Cyclopropanecarboxamide & $\mathrm{C}_{4} \mathrm{H}_{7} \mathrm{NO}$ & 85.1 & No activity \\
\hline 4 & 4.24 & 2.36 & 5-Amino-6-nitrosopyrimidine-2,4(1h,3h)-dione & $\mathrm{C}_{4} \mathrm{H}_{4} \mathrm{~N}_{4} \mathrm{O}_{3}$ & 156.1 & Antibacterial \\
\hline 5 & 4.47 & 0.25 & Hexyl octanoate & $\mathrm{C}_{14} \mathrm{H}_{28} \mathrm{O}_{2}$ & 228.4 & Flavouring agent \\
\hline 6 & 4.54 & 1.43 & Pyrrolidin-5-one, 2,3-dedihydro-3-nitro- & $\mathrm{C}_{4} \mathrm{H}_{3} \mathrm{NO}_{4}$ & 129.1 & No activity \\
\hline 7 & 4.69 & 0.27 & 3-Amino-2-oxazolidinone & $\mathrm{C}_{3} \mathrm{H}_{6} \mathrm{~N}_{2} \mathrm{O}_{2}$ & 102.1 & No activity \\
\hline 8 & 4.76 & 3.70 & $\begin{array}{l}\text { 4H-Pyran-4-one, } \\
\text { 2,3-dihydro-3,5-dihydroxy-6-methyl- }\end{array}$ & $\mathrm{C}_{6} \mathrm{H}_{8} \mathrm{O}_{4}$ & 144.1 & $\begin{array}{c}\text { Antibacterial, } \\
\text { anti-inflammatory, } \\
\text { antiproliferative antioxidant, } \\
\text { automatic nerve activity, } \\
\text { anticancer }\end{array}$ \\
\hline 9 & 5.13 & 0.84 & 3,4-Pentadienal & $\mathrm{C}_{5} \mathrm{H}_{6} \mathrm{O}$ & 82.1 & No activity \\
\hline 10 & 5.27 & 0.34 & cyclobut-1-enylmethanol & $\mathrm{C}_{5} \mathrm{H}_{8} \mathrm{O}$ & 84.1 & Antibacterial \\
\hline 11 & 5.32 & 7.20 & 5-Hydroxymethylfurfural & $\mathrm{C}_{6} \mathrm{H}_{6} \mathrm{O}_{3}$ & 126.1 & $\begin{array}{l}\text { Anti-oxidative, anti-allergic, } \\
\text { anti-inflammatory, } \\
\text { anti-hypoxic, } \\
\text { anti-hyperuricemic }\end{array}$ \\
\hline 12 & 5.89 & 0.21 & 6-O-Acetyl-beta-D-glucose- & $\mathrm{C}_{8} \mathrm{H}_{14} \mathrm{O}_{7}$ & 222.2 & \\
\hline 13 & 5.93 & 0.43 & $\begin{array}{l}\text { 6-Oxa-1-azabicyclo(3.1.0)hexane, } \\
\text { 2,2-dimethyl-4,5-diphenyl-, trans- }\end{array}$ & $\mathrm{C}_{18} \mathrm{H}_{19} \mathrm{NO}$ & 265.3 & Antibacterial \\
\hline 14 & 6.12 & 0.62 & $\mathrm{~N}$-(2-Methoxyethyl)alanine & $\mathrm{C}_{6} \mathrm{H}_{13} \mathrm{NO}_{3}$ & 147.2 & No activity \\
\hline 15 & 6.17 & 1.00 & trans-2-Butenyl acetate & $\mathrm{C}_{6} \mathrm{H}_{10} \mathrm{O}_{2}$ & & No activity \\
\hline 16 & 6.81 & 0.96 & N-Acetyl-d-serine & $\mathrm{C}_{5} \mathrm{H}_{9} \mathrm{NO}_{4}$ & 147.1 & No activity \\
\hline 17 & 6.84 & 1.03 & $\begin{array}{l}\text { alpha-D-Mannopyranoside, methyl } \\
\text { 3,6-anhydro- }\end{array}$ & $\mathrm{C}_{7} \mathrm{H}_{12} \mathrm{O}_{5}$ & 176.2 & Antibacterial \\
\hline 18 & 7.19 & 0.27 & $\begin{array}{l}\text { 2(4H)-Benzofuranone, } \\
\text { 5,6,7,7a-tetrahydro-6-hydroxy-4,4,7a-trimethyl- }\end{array}$ & $\mathrm{C}_{11} \mathrm{H}_{16} \mathrm{O}_{3}$ & 196.2 & Antibacterial \\
\hline 19 & 7.24 & 0.46 & 2-Mercaptopyridine-4-ol & $\mathrm{C}_{5} \mathrm{H}_{5} \mathrm{NOS}$ & 127.17 & No activity \\
\hline 20 & 7.71 & 3.5 & $\begin{array}{l}\text { 1,3,4,5-Tetrahydroxycyclohexanecarboxylic } \\
\text { acid }\end{array}$ & $\mathrm{C}_{7} \mathrm{H}_{12} \mathrm{O}_{6}$ & 192.2 & Hepatoprotective \\
\hline 21 & 8.15 & 1.00 & d-Glycero-d-galacto-heptose & $\mathrm{C}_{7} \mathrm{H}_{14} \mathrm{O}_{7}$ & 210.1 & Antioxidant \\
\hline 22 & 8.27 & 0.30 & 7-Hydroxy-3-(1,1-dimethylprop-2-enyl)coumarin & $\mathrm{C}_{14} \mathrm{H}_{14} \mathrm{O}_{3}$ & 230.3 & Antibacterial, antitumor \\
\hline 23 & 8.30 & 1.11 & N-Acetyl-D-Glucosamine & $\mathrm{C}_{8} \mathrm{H}_{15} \mathrm{NO}_{6}$ & 221.1 & $\begin{array}{l}\text { Antibacterial, antitumor, } \\
\text { antioxidant, anticoagulant, } \\
\text { wound healing }\end{array}$ \\
\hline 24 & 8.39 & 0.64 & Z-8-Methyl-9-tetradecen-1-ol acetate & $\mathrm{C}_{17} \mathrm{H}_{32} \mathrm{O}_{2}$ & 268.4 & No activity \\
\hline 25 & 8.5 & 0.21 & $\begin{array}{l}\text { 3-Methyl-4-(phenylthio)-2-prop-2-enyl-2,5 } \\
\text {-dihydrothiophene 1,1-dioxide }\end{array}$ & $\mathrm{C}_{14} \mathrm{H}_{16} \mathrm{O}_{2} \mathrm{~S}_{2}$ & 280.4 & No activity \\
\hline 26 & 8.75 & 0.69 & Methyl tricosanoate & $\mathrm{C}_{24} \mathrm{H}_{48} \mathrm{O}_{2}$ & 368.6 & Antibacterial \\
\hline 27 & 8.93 & 2.05 & Palmitic acid & $\mathrm{C}_{6} \mathrm{H}_{32} \mathrm{O}_{2}$ & 256.4 & $\begin{array}{l}\text { Antioxidant, antitumor, } \\
\text { anti-inflammatory, } \\
\text { antibacterial, antifungal }\end{array}$ \\
\hline 28 & 9.14 & 0.77 & Pentadecanoic acid & $\mathrm{C}_{15} \mathrm{H}_{30} \mathrm{O}_{2}$ & 242.4 & $\begin{array}{c}\text { Adhesives, agricultural } \\
\text { chemicals (non-pesticidal), } \\
\text { lubricants }\end{array}$ \\
\hline 29 & 9.19 & 0.41 & 26-Hydroxycholesterol & $\mathrm{C}_{27} \mathrm{H}_{46} \mathrm{O}_{2}$ & 402.7 & No activity \\
\hline 30 & 9.26 & 0.50 & Hexacosanoic acid & $\mathrm{C}_{6}{ }_{6} \mathrm{H}_{52} \mathrm{O}_{2}$ & 396.7 & No activity \\
\hline 31 & 9.32 & 0.60 & Palmitic acid & $\mathrm{C}_{6} \mathrm{H}_{32} \mathrm{O}_{2}$ & 256.4 & $\begin{array}{l}\text { Antioxidant, antitumor, } \\
\text { anti-inflammatory, } \\
\text { antibacterial, antifungal }\end{array}$ \\
\hline 32 & 9.43 & 1.78 & 9,12,15-Octadecatrienoic acid, methyl ester & $\mathrm{C}_{16} \mathrm{H}_{32} \mathrm{O}_{2}$ & 292.5 & No activity \\
\hline 33 & 9.47 & 1.24 & Phytol & $\mathrm{C}_{20} \mathrm{H}_{40} \mathrm{O}$ & 296.5 & $\begin{array}{c}\text { Antioxidant, analgesic, } \\
\text { antibacterial, } \\
\text { anti-inflammatory, anticancer, } \\
\text { and neuroprotective }\end{array}$ \\
\hline 34 & 9.61 & 2.96 & $1,5,9,13$-Tetradecatetraene & $\mathrm{C}_{14} \mathrm{H}_{22}$ & 190.3 & No activity \\
\hline 35 & 9.80 & 3.40 & 2-Linoleoylglycerol & $\mathrm{C}_{21} \mathrm{H}_{38} \mathrm{O}_{4}$ & 354.5 & Antibacterial \\
\hline 36 & 9.94 & 0.42 & Stearic acid & $\mathrm{C}_{18} \mathrm{H}_{36} \mathrm{O}_{2}$ & 284.5 & Antioxidant, antibacterial \\
\hline 37 & 9.98 & 0.50 & Epoxycholesterol & $\mathrm{C}_{27} \mathrm{H}_{46} \mathrm{O}_{2}$ & 402.7 & No activity \\
\hline 38 & 10.06 & 3.06 & Hexadecanoate & $\mathrm{C}_{16} \mathrm{H}_{31} \mathrm{O}_{2}$ & 255.42 & Anti-inflammatory \\
\hline 39 & 10.21 & 1.56 & 4alpha,5alpha-Epoxycholestane & $\mathrm{C}_{27} \mathrm{H}_{46} \mathrm{O}$ & 386.7 & No activity \\
\hline 40 & 10.70 & 2.83 & 6,10,14-Trimethylpentadecan-2-one & $\mathrm{C}_{18} \mathrm{H}_{36} \mathrm{O}$ & 268.5 & antibacterial \\
\hline 41 & 10.80 & 1.05 & 1-Cinnamyl-3-methylindole-2-carbaldehyde & $\mathrm{C}_{19} \mathrm{H}_{17} \mathrm{NO}$ & 275.1 & Antioxidant, antibacterial \\
\hline 42 & 10.85 & 0.92 & 3,5-Bis-(trichloromethyl)-benzoyl chloride & $\mathrm{C}_{9} \mathrm{H}_{3} \mathrm{Cl}_{7} \mathrm{O}$ & 375.3 & No activity \\
\hline
\end{tabular}


Table 1. Cont.

\begin{tabular}{|c|c|c|c|c|c|c|}
\hline S/L no & R. Time (min) & PA (\%) & Compound Name & Molecular Formula & MW (g/mol) & *** Activity \\
\hline \multicolumn{7}{|c|}{ Leaves } \\
\hline 43 & 10.92 & 5.44 & 2-Stearoylglycerol & $\mathrm{C}_{21} \mathrm{H}_{42} \mathrm{O}_{4}$ & 358.6 & No activity \\
\hline 44 & 11.60 & 1.58 & $\begin{array}{l}\text { Tricyclo[10.2.2.2(5,8)]octadeca- } \\
5,7,12,14,15,17 \text {-hexaene }\end{array}$ & $\mathrm{C}_{18} \mathrm{H}_{20}$ & 236.3 & Antifungal \\
\hline 45 & 11.70 & 2.17 & 9,12,15-Octadecatrien-1-ol & $\mathrm{C}_{18} \mathrm{H}_{32} \mathrm{O}$ & 264.4 & Antioxidant, antibacterial \\
\hline 46 & 11.74 & 4.50 & 1,5,9,13-Tetradecatetraene & $\mathrm{C}_{14} \mathrm{H}_{22}$ & 190.3 & No activity \\
\hline 47 & 11.79 & 3.16 & 5 beta-Coprostanol & $\mathrm{C}_{27} \mathrm{H}_{48} \mathrm{O}$ & 388.7 & No activity \\
\hline 48 & 12.24 & 1.64 & $\begin{array}{l}\text { Curan-17-oic acid, } \\
\text { 2,16-didehydro-20-hydroxy-19-oxo-, } \\
\text { methyl ester }\end{array}$ & $\mathrm{C}_{20} \mathrm{H}_{22} \mathrm{~N}_{2} \mathrm{O}_{4}$ & 354.4 & Antibacterial, antifungal \\
\hline 49 & 14.65 & 4.45 & Vitamin E & $\mathrm{C}_{29} \mathrm{H}_{50} \mathrm{O}_{2}$ & 430.7 & $\begin{array}{c}\text { Antioxidant, antibacterial, } \\
\text { Analgesic, anti-inflammatory, } \\
\text { anxiolytic and } \\
\text { antidepressant, }\end{array}$ \\
\hline 50 & 15.28 & 1.52 & $\begin{array}{l}\text { 3-\{[(3,5-Dichlorophenyl)imino]methyl\}- } \\
\text { 1,2-benzenediol }\end{array}$ & $\mathrm{C}_{13} \mathrm{H}_{9} \mathrm{Cl}_{2} \mathrm{NO}_{2}$ & 282.1 & Antibacterial, antifungal \\
\hline 51 & 16.05 & 0.85 & $\begin{array}{l}\text { Methyl 4-oxo-4,5,6,7-tetrahydro- } \\
\text { 1H-indole-2-carboxylate }\end{array}$ & $\mathrm{C}_{10} \mathrm{H}_{11} \mathrm{NO}_{3}$ & 193.2 & Antidiabetic \\
\hline 52 & 16.26 & 1.07 & Stigmasta-5,22-dien-3-ol & $\mathrm{C}_{29} \mathrm{H}_{48} \mathrm{O}$ & 412.7 & Antibacterial \\
\hline 53 & 17.05 & 3.93 & Clionasterol & $\mathrm{C}_{29} \mathrm{H}_{50} \mathrm{O}$ & 414.7 & Antibacterial \\
\hline 54 & 17.81 & 1.71 & Hexadecahydropyrene & $\mathrm{C}_{16} \mathrm{H}_{26}$ & 218.3 & No activity \\
\hline 55 & 18.56 & 3.76 & alpha-Amyrin & $\mathrm{C}_{30} \mathrm{H}_{50} \mathrm{O}$ & 426.7 & $\begin{array}{l}\text { Analgesic, anti-inflammatory, } \\
\text { anxiolytic and antidepressant }\end{array}$ \\
\hline \multicolumn{7}{|c|}{ Seed } \\
\hline 1 & 4.00 & 0.92 & Glycerine & $\mathrm{C}_{3} \mathrm{H}_{8} \mathrm{O}_{3}$ & 92.1 & Antibacterial \\
\hline 2 & 5.29 & 0.03 & Benzofuran, 2, 3-dihydro- & $\mathrm{C}_{15} \mathrm{H}_{14} \mathrm{OS}$ & 242.3 & $\begin{array}{l}\text { Antidepressant, anticancer, } \\
\text { antiviral, antifungal, } \\
\text { antioxidant, anti-psychotic }\end{array}$ \\
\hline 3 & 6.89 & 0.16 & N-Acetylethylenediamine & $\mathrm{C}_{4} \mathrm{H}_{10} \mathrm{NO}_{2}$ & 102.1 & Antibacterial \\
\hline 4 & 7.76 & 0.08 & d-Mannitol, 1, 4-anhydro- & $\mathrm{C}_{6} \mathrm{H}_{12} \mathrm{O}_{5}$ & 164.2 & No activity \\
\hline 5 & 8.08 & 0.03 & Octadecanoic acid & $\mathrm{C}_{18} \mathrm{H}_{36} \mathrm{O}_{2}$ & 284.5 & Antibacterial \\
\hline 6 & 8.75 & 0.05 & Hexadecanoic acid, methyl ester & $\mathrm{C}_{17} \mathrm{H}_{34} \mathrm{O}_{2}$ & 270.5 & $\begin{array}{l}\text { Antibacterial, anticancer, } \\
\text { anti-inflammatory, } \\
\text { anti-diuretic }\end{array}$ \\
\hline 7 & 9.05 & 8.83 & Hexadecanoic acid & $\mathrm{C}_{16} \mathrm{H}_{32} \mathrm{O}_{2}$ & 256.4 & $\begin{array}{l}\text { Antioxidant, antitumor, } \\
\text { anti-inflammatory, } \\
\text { antibacterial, antifungal }\end{array}$ \\
\hline 8 & 9.43 & 1.42 & Hexadecanoic acid & $\mathrm{C}_{16} \mathrm{H}_{32} \mathrm{O}_{2}$ & 256.4 & $\begin{array}{l}\text { Antioxidant, antitumor, } \\
\text { anti-inflammatory, } \\
\text { antibacterial, antifungal }\end{array}$ \\
\hline 9 & 9.81 & 77.46 & 9-octadecanoic acid (z)- & $\mathrm{C}_{18} \mathrm{H}_{34} \mathrm{O}_{2}$ & 282.5 & Antibacterial, antifungal \\
\hline 10 & 10.71 & 1.58 & Linoleic acid & $\mathrm{C}_{18} \mathrm{H}_{32} \mathrm{O}_{2}$ & 280.4 & Antibacterial \\
\hline 11 & 10.95 & 2.85 & Linoleic acid & $\mathrm{C}_{18} \mathrm{H}_{32} \mathrm{O}_{2}$ & 280.4 & Antibacterial \\
\hline 12 & 11.75 & 6.21 & $\begin{array}{c}\text { 9, 12-octadecadienoic acid ( } \mathrm{z}, \mathrm{z}) \text {-, } \\
\text { 2-hydroxy-1-(hydroxymethyl)ethyl ester }\end{array}$ & $\mathrm{C}_{21} \mathrm{H}_{38} \mathrm{O}_{4}$ & 354.5 & Antioxidant, antibacterial \\
\hline 13 & 13.97 & 0.33 & $\begin{array}{c}\text { 17-(acetyloxy)-4, } \\
\text { 4-dimethyl-7-oxoandrost-5-en-3-yl acetate }\end{array}$ & $\mathrm{C}_{25} \mathrm{H}_{36} \mathrm{O}_{5}$ & 416.5 & No activity \\
\hline 14 & 17.07 & 0.04 & .beta.-Sitosterol & $\mathrm{C}_{29} \mathrm{H}_{50} \mathrm{O}$ & 414.7 & Antidiabetic, antibacterial \\
\hline
\end{tabular}

SL no: serial number, R.T: retention time, PA: peak area, MW: molecular weight, ${ }^{* * *}$ Activity Source: Dr. Duke's phytochemical and ethno-botanical databases [30].

\subsection{Total Phenol and Flavonoid Content}

Four different extracts (NHX, EA, ETH, and WT) of Kenaf leaves and seed were analyzed to quantify phenolic and flavonoid content. The phenolic content was assessed by gallic acid and expressed as GAE per $100 \mathrm{~g}$ of dry extract (Figure 3A), whereas flavonoid content was estimated by quercetin and expressed as QE per $100 \mathrm{~g}$ of dry extract (Figure 3B). The highest amount of phenolic and flavonoid content was recorded in seed compared to leaves. Particularly, WT extract demonstrated the highest total phenol content for both Kenaf seed $(754.6 \pm 3.14 \mathrm{mg} / 100 \mathrm{~g}$ dry extract) and leaves $(418.7 \pm 3.47 \mathrm{mg} / 100 \mathrm{~g}$ dry extract). Similarly, the maximum flavonoid content was also detected from the WT $(425.33 \pm 4.39$ and $299.17 \pm 3.43 \mathrm{mg} / 100 \mathrm{~g}$ dry extract $)$ extract of both Kenaf seed and leaves. However, the other solvent seed extracts of EA followed by ETH and NHX, as well as leaf extracts of ETH, followed by EA and NHX were noted as significant for both TPC and TFC. 
The highest TPC and TFC observed in the water extract might have resulted from its high polarity index [31]. The important phytoconstituents of a plant can be polar or nonpolar in nature-mainly, phenolic compounds possess abundant hydroxyl groups, which are responsible for dissolving of the polar solvent [32]. In addition, the ethyl acetate extract displayed better extraction of TPC and TFC, which confirmed that this solvent is also efficient for extracting secondary metabolites. It is stated that the ethyl acetate solvent has potential for the extraction of phenolic compounds, and this statement is in agreement with the result found from the EA extract of Kenaf seed [33]. Besides, noteworthy TPC and TFC manifested by the ethanol extract might be owing to the presence of methyl radicles, which can easily conjugate with phenolic or flavonoid compounds and allow efficient solvation [34]. In contrast, hexane showed poor extraction of TPC and TFC, which could be attributed to the lower polarity or strong non-polar nature of the solvent [35].
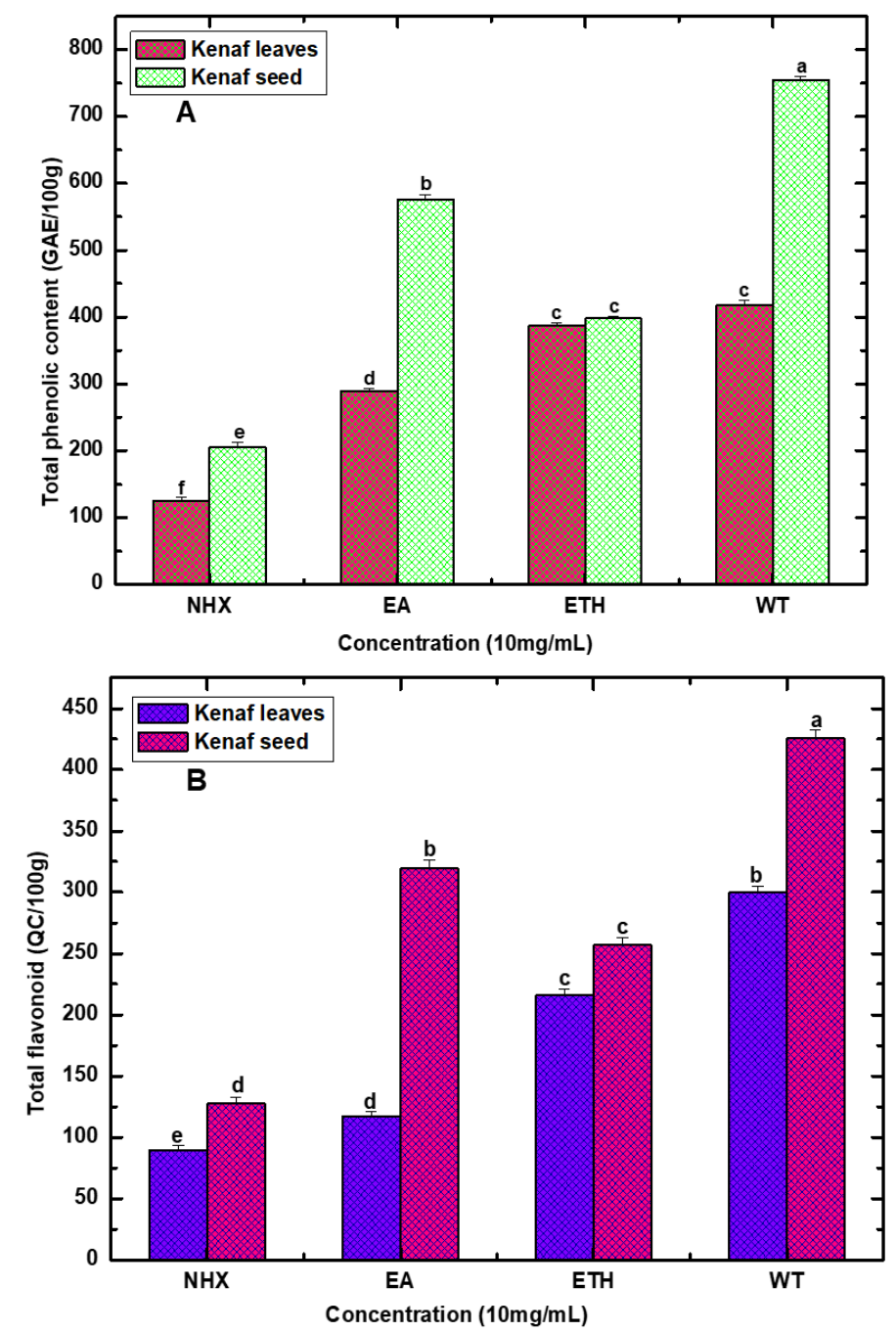

Figure 3. Total phenolic (A) and flavonoid (B) content of various solvent extracts (NHX, EA, ETH, and WT) of Kenaf leaves and seed. Values are expressed as mean $\pm \mathrm{SD}(\mathrm{n}=3)$. Values marked by different letters in each column are significantly different by t-test $(p<0.05)$. GAE = gallic acid equivalent, $\mathrm{QC}=$ quercetin equivalent, $\mathrm{NHX}=n$-Hexane extract, $\mathrm{EA}=$ Ethyl acetate extract, $\mathrm{ETH}=$ Ethanol extract, and $\mathrm{WT}=$ Water extract.

\subsection{Antioxidant Activity}

Figure 4A,B displays the percentage inhibition of the DPPH and $\mathrm{OH}$ free radical scavenging capacity of Kenaf leaves and seed extracts. The activity notably varied among the various extracts, 
and the overall higher percentage of scavenging capacity was observed in the seed extracts compared to leaves. In both cases, the antioxidant capacity of ethanol and ethyl acetate extract was almost identical; even no noticeable changes in regard to activity were noted for the DPPH and $\mathrm{H}_{2} \mathrm{O}_{2}$ free radical scavenging. However, in the case of DPPH, the highest antioxidant activity of Kenaf seed was recorded for WT $(73.12 \pm 2.06 \%)$, followed by ethyl acetate $(54.45 \pm 1.38 \%)$, ETH $(52.49 \pm 1.12 \%)$, and hexane $(28.09 \pm 2.61 \%)$ extract. However, Kenaf leaves also exhibited $65.35 \pm 1.86 \%$ DPPH scavenging for the WT, $46.27 \pm 1.71 \%$ (EHT), $43.19 \pm 2.15 \%$ (EA), and $21.07 \pm 1.65 \%$ (NHX) extracts. These findings were analogous with many previous studies, where authors concluded that relatively high polar solvent extracts, such as water, methanol, and ethanol exposed higher DPPH free radical potential than the non-polar solvent extracts. Likewise, DPPH, a similar pattern of antioxidant activity, was observed in the case of the $\mathrm{H}_{2} \mathrm{O}_{2}$ scavenging test. Here, Kenaf seed extracts demonstrated strong hydroxyl radical scavenging activity, ranging from $67.69 \pm 1.46$ to $26.41 \pm 2.58 \%$, whereas leaf extracts manifested between $53.86 \pm 0.43$ and $19.52 \pm 1.83 \%$.
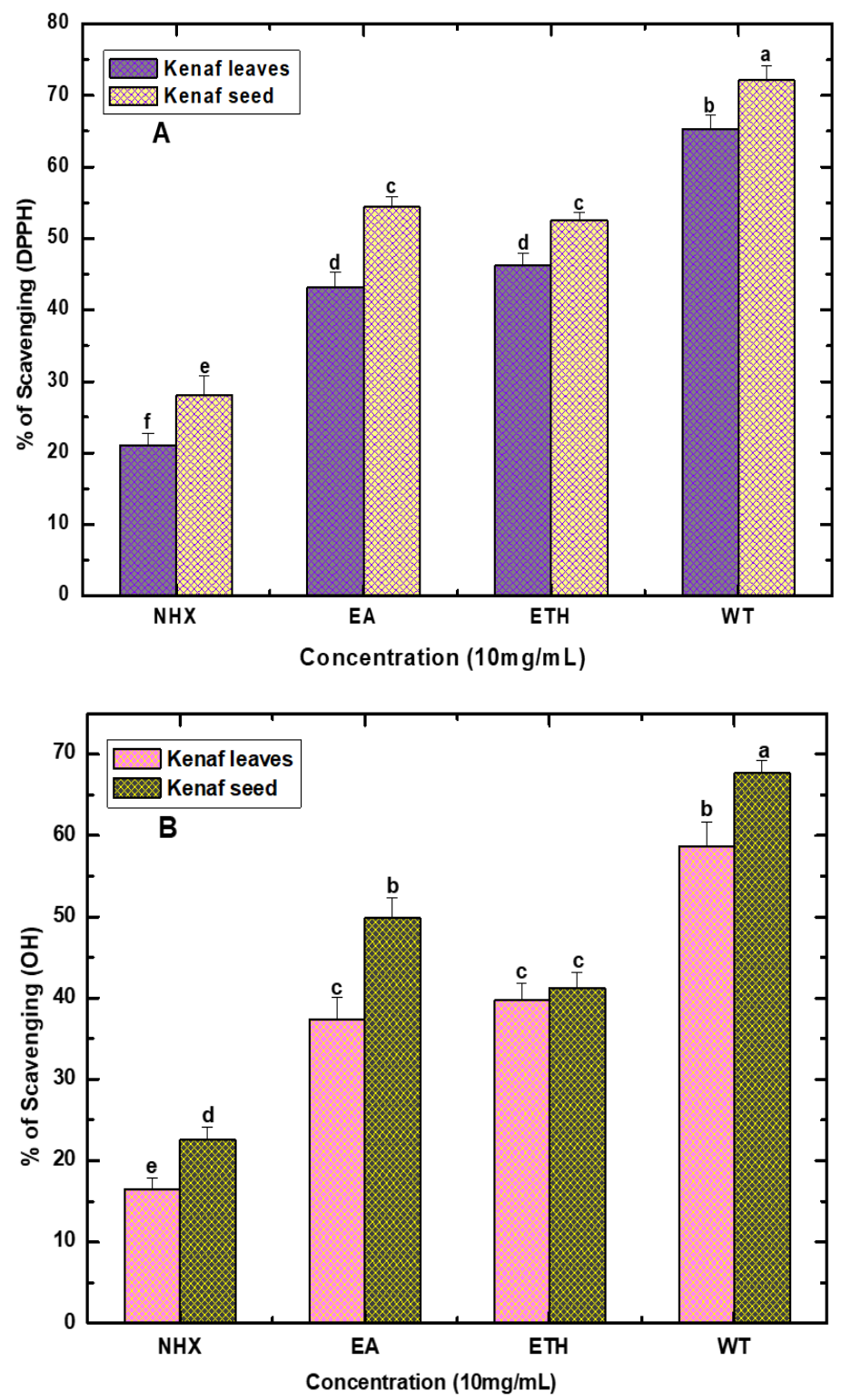

Figure 4. DPPH (A) and hydrogen peroxide (B) scavenging capacity of various solvent extracts (NHX, EA, ETH, and WT) of Kenaf leaves and seed. Values are expressed as mean $\pm \mathrm{SD}(\mathrm{n}=3)$. Values marked by different letters in each column are significantly different by $\mathrm{t}$-test $(p<0.05)$. NHX $=n$-Hexane extract, EA = Ethyl acetate extract, ETH $=$ Ethanol extract, and WT $=$ Water extract. 
Interestingly, an interplay relationship exists between antioxidant activity and the quantity of secondary metabolites [36]. Usually, phenolics are regarded as the predominant antioxidant components, and scavenging activities of these components are directly proportional to the total content of phenolics [18]. Hence, the observed higher antioxidant activity in our study might be attributed to secondary metabolites, such as higher content of polyphenol and flavonoids of the extracts. Nevertheless, these outcomes indicate that this plant has remarkable scavenging capacity.

\subsection{Cytotoxicity and Anti-Lung Cancer Activity}

The cytotoxicity of the Kenaf leaf and seed extracts was determined against NIH3T3 cells. Figure 5A displays the Kenaf seed extracts which expressed slight toxicity with increasing concentrations in a dose-dependent fashion. Notably, the hexane and ethyl acetate extracts showed higher cytotoxicity of $23.64 \pm 0.12 \%$ and $18.77 \pm 0.13 \%$, respectively, and cell growth inhibition was detected at a higher concentration $(1000 \mu \mathrm{g} / \mathrm{mL})$. In the case of Kenaf leaves (Figure $5 \mathrm{~B})$, a similar cytotoxicity pattern was also exposed by the hexane and ethyl acetate extracts. Here, cell growth inhibition was recorded as $16.37 \pm 0.20 \%$ and $12.7 \pm 0.37 \%$, respectively, at the same concentration. However, treatment of water and ethanol extracts (both seed and leaves) did not reveal any obvious toxicity toward the NIH3T3 cells, even at the higher concentration treatment, which indicates that these extracts are comparatively safe than the others. The observed healthy cells after the treatment of water and ethanol extract might be caused by their higher content of polyphenol, flavonoid, and promising antioxidant activity, which acted as nutrients for the cell growth [37]. In contrast, the toxicity demonstrated by hexane extract might be attributed to its poor antioxidant capacity, which affected the cells by producing reactive oxygen species [38].

Figure 6A,B displays the anti-lung cancer activity of Kenaf seed and leaves against A549 cells. Here, NHX extract exposed the highest anti-lung cancer activity in a concentration-dependent response, where treatment of the optimal concentration $(1000 \mu \mathrm{g} / \mathrm{mL})$ revealed $37.4 \%$ cell death by Kenaf seed and $29.6 \%$ by Kenaf leaves. The comparable accord was also observed for ETH and EA extract. In Kenaf seed, cell viability was reduced to $33.8 \%$ by ETH, while EA decreased $29.7 \%$ cell viability at the highest concentration. In contrast, ETH (26.4\%) and EA (23.6\%) of Kenaf leaves also manifested moderate anticancer activity. Interestingly, NHX demonstrated toxicity against NIH3T3 cells, and ETH was found to be safe for NIH3T3 cells; however, during an anti-lung cancer test, both extracts exhibited remarkable inhibition of A549 cell growth, which concludes that the ETH extract is relatively safer than the other extracts. Previously, a study conducted by Wong et al. revealed that the optimum concentration of Kenaf seed extract strongly inhibited the cell growth of HeLa (CCL-2), breast cancer (MCF-7), colon cancer (HCT-116), and lung cancer (SK-LU1) [39], which also support our findings. Importantly, the treatment of 5\% DMSO in the NIH3T3 and A549 cells did not expose any notable toxicity, wherein $90 \%$ of cell confluence was observed (visual observation), which suggests that the solvent (5\% DMSO) did not have a toxicological effect. 

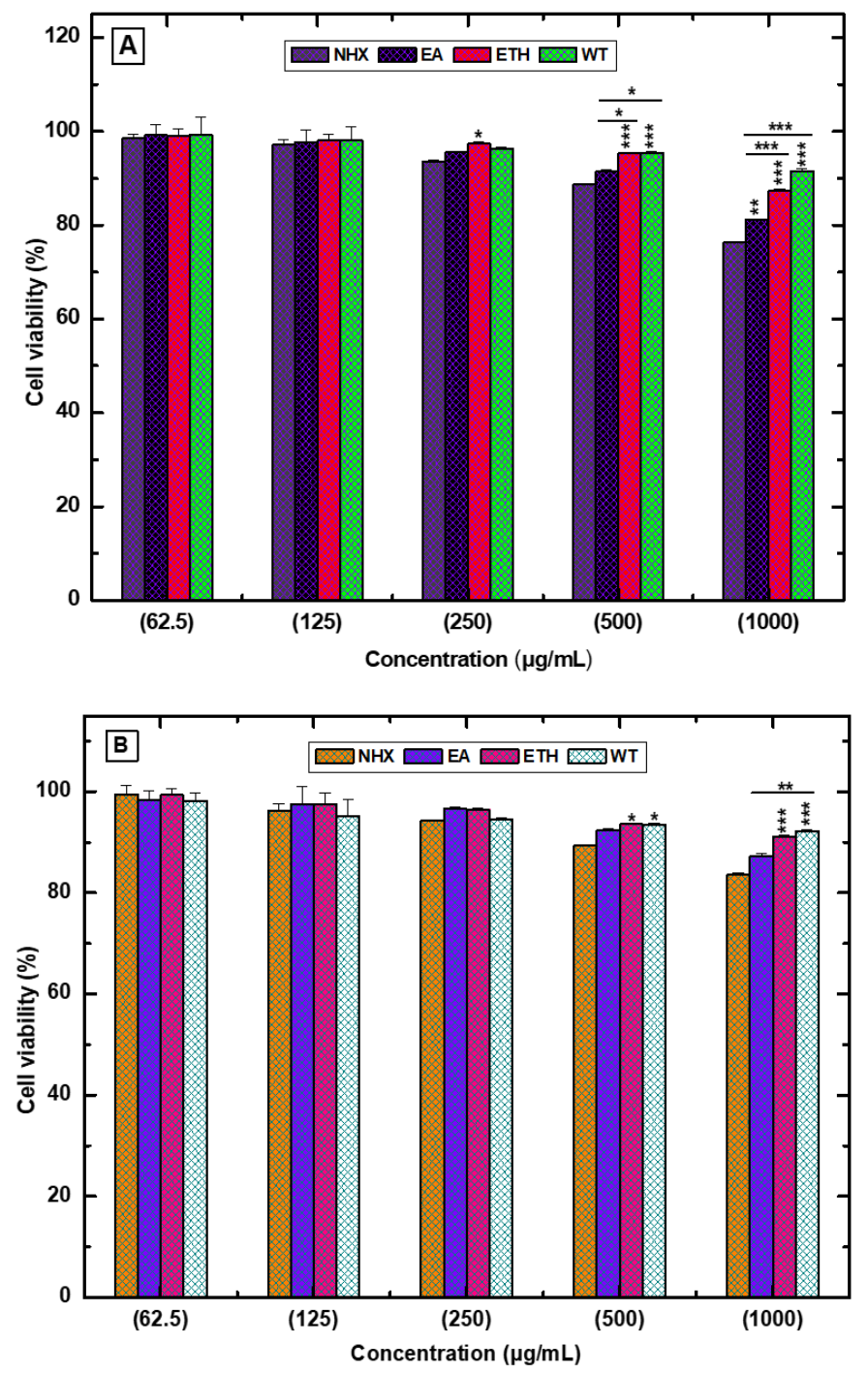

Figure 5. Cytotoxicity of various solvent extracts (NHX, EA, ETH, and WT) of Kenaf seed (A) and leaves $(\mathbf{B})$. Here, NHX $=n$-Hexane extract, EA $=$ Ethyl acetate extract, $\mathrm{ETH}=$ Ethanol extract, and $\mathrm{WT}=$ Water extract. Data was analyzed using GraphPad Prism 6.0 statistical software. Values are expressed as mean $\pm S D(n=3)$, and a two-way ANOVA andBonferroni test were applied. They were significantly different when comparing each column to all other columns at ${ }^{*} p<0.05,{ }^{* *} p<0.01$, and ${ }^{* * *} p<0.001$.
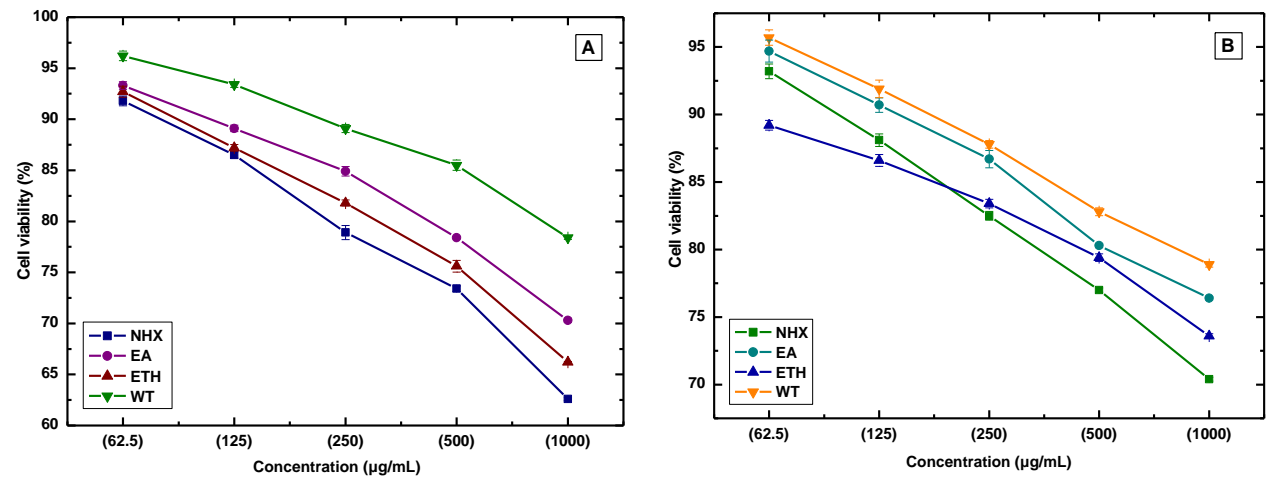

Figure 6. Anti-lung cancer activity of various solvent extracts (NHX, EA, ETH, and WT) of Kenaf seed (A) and leaves (B). Here, NHX $=n$-Hexane extract, EA $=$ Ethyl acetate extract, ETH $=$ Ethanol extract, and $\mathrm{WT}=$ Water extract. Values are expressed as mean $\pm \mathrm{SD}(\mathrm{n}=3)$. 
Previously, Kenaf seed-mediated silver nanoparticles showed promising anti-lung cancer activity [15]. It was reported that the plant possesses bioactive compounds which induce mitochondrial damage by elevating the superoxide level that suppresses cancer cell growth through reduction of ATP synthesis [40]. Some secondary metabolites may also attack the DNA, resulting in abundant ROS production and apoptosis-inducing enzyme activation, subsequently leading to cell death [41].

\subsection{Antibacterial Activity}

The antibacterial activity of Kenaf leaves and seed extracts are presented in Table 2. Both Gram-positive (Staphylococcus aureus, Bacillus cereus, and Bacillus subtilis) and Gram-negative (Salmonella Typhi, Escherichia coli, and Pseudomonas aeruginosa) microorganisms were tested through the disc diffusion method. Results revealed that Kenaf seed extracts were very effective against both Gram-positive and Gram-negative bacteria. Ethyl acetate, ethanol, and water extract exposed antibacterial activity against B. cereus, E. coli, and B. subtilis microorganisms. Here, the most remarkable inhibitory effect was observed against $E$. coli bacteria, where the zone of inhibition was recorded for $15.2 \pm 0.72 \mathrm{~mm}$ for ethyl acetate extract. In the case of leaf extracts, the zone of inhibition recorded for ETH and WT were the most potent among all other extracts. These results indicate that the Kenaf plant possesses a broad spectrum antipathogenic effect. Moreover, both leaves and seed extracts of NHX did not show any inhibitory effect against any bacteria. On the other hand, both leaf and seed extracts did not manifest any activity against Gram-positive (Staphylococcus aureus) and Gram-negative (Salmonella Typhi and Pseudomonas aeruginosa) microorganisms. In the case of the negative control (5\% DMSO), no activity was recorded, which indicates that the 5\% DMSO did not have an influence on the extract's activity.

Table 2. Antibacterial effects of various solvent extracts of the Kenaf leaf and seed.

\begin{tabular}{|c|c|c|c|c|c|c|}
\hline & & & \multicolumn{4}{|c|}{ Zone of Inhibition (mm) } \\
\hline Bacterial Strain & \multirow{2}{*}{\multicolumn{2}{|c|}{$\begin{array}{l}\text { Gram Negative } \\
\text { Escherichia Coli }\end{array}$}} & \multicolumn{4}{|c|}{ Gram Positive } \\
\hline \multirow{2}{*}{$\begin{array}{c}\text { Name of Bacteria } \\
\text { Concentration }(50 \mathrm{mg} / \mathrm{mL})\end{array}$} & & & \multicolumn{2}{|c|}{ Bacillus Cereus } & \multicolumn{2}{|c|}{ Bacillus Subtilis } \\
\hline & Leaves & Seed & Leaves & Seed & Leaves & Seed \\
\hline NHX & - & - & - & - & - & - \\
\hline EA & - & $15.2 \pm 0.72$ & - & $12.8 \pm 0.78$ & - & $11.6 \pm 0.59$ \\
\hline ETH & $9.8 \pm 1.41$ & $13.1 \pm 0.34$ & - & $11.2 \pm 0.37$ & - & $10.1 \pm 0.41$ \\
\hline WT & $11.3 \pm 0.38$ & $13.7 \pm 0.56$ & $9.7 \pm 0.73$ & $12.5 \pm 0.82$ & - & $9.3 \pm 0.28$ \\
\hline Standard Ampicillin (25 ug/mL) & $29.4 \pm 0.26$ & $27.9 \pm 0.48$ & $26.4 \pm 0.52$ & $28.4 \pm 0.47$ & $30.1 \pm 0.18$ & $28.4 \pm 0.47$ \\
\hline
\end{tabular}

Values are presented as mean inhibition zone $(\mathrm{mm}) \pm \mathrm{SD}$ of three replicates, $-:$ no activity, NHX $=n$-Hexane extract, $\mathrm{EA}=$ Ethyl acetate extract , and $\mathrm{WT}=$ Water extract.

The observed antibacterial efficiencies of the Kenaf plant might be due to the higher content of phenols and flavonoids [42]. It is stated that the secondary metabolites present in the plant are the key source of diverse pharmacological actions [43]. These metabolites also provide natural defensive pathways to inhibit various insects and pathogens, such as viruses and fungi [44]. In addition, GC-MS analysis of Kenaf leaves and seed exposed several potential bioactive constituents, which might play a crucial role either by interrupting normal cellular functions [45] or destabilizing the bacterial membrane [46].

\section{Conclusions}

This research explored the various solvent extractions of Kenaf leaves and seed and their pharmacological potentials. Among the solvents tested, ethanol was the efficient solvent, and demonstrated the highest extraction yield and potential bioactive compounds. In contrast, the water solvent remarkably influenced phytochemical content. In the case of leaves and seed, water extracts significantly impacted on the recovery of the highest total polyphenol, flavonoids, and manifestation of noteworthy antioxidant activity. In vitro cytotoxic activity revealed that extracts of Kenaf leaves and seed were non-toxic to the healthy (NIH3T3) cells, except $n$-hexane extracts, which expressed slight toxicity. Besides, $n$-hexane and ethanol extracts manifested promising anti-lung cancer activity 
against A549 cells, where ethanol extracts were comparatively safer than other extracts. During the antibacterial test, extracts of Kenaf seed exhibited significant activity against Gram-positive and Gram-negative microorganisms, as evidenced by the notable zone of inhibition. This work collectively concluded that Kenaf seed extracts was found very potent in terms of important bioactive compounds and pharmacological aspects, which can be an excellent biological matrix of natural antioxidants.

Author Contributions: M.A. and K.K.O., prepared the extracts, carried out all the experimental works and collected and analyzed the data. M.A. conceived and designed the experiments and drafted the final manuscript. M.O.K.A. and M.H.S. revised the manuscript. M.-H.W. co supervised this study. D.H.C. supervised the study. All authors have read and agreed to the published version of the manuscript.

Funding: This research did not receive any specific grant from funding agencies in the public, commercial, or not-for-profit sectors.

Acknowledgments: This research was acknowledged by the Kangwon National University, Chuncheon 24341, Korea.

Conflicts of Interest: The authors declare that they have no conflict of interest.

\section{References}

1. Young, I.S.; Woodside, J.V. Antioxidants in health and disease. J. Clin. Pathol. 2001, 54, 176-186. [CrossRef]

2. Harman, D. Aging: Phenomena and theories. Ann. N. Y. Acad. Sci. 1998, 854, 1-7. [CrossRef] [PubMed]

3. Adnan, M.; Mohammad, K.I.; Manik, M.E.H. Anticancer agents in combination with statins. J. Bioequiv. Availab. 2017, 9, 463-466. [CrossRef]

4. Anagnostopoulou, M.A.; Kefalas, P.; Papageorgiou, V.P.; Assimopoulou, A.N.; Boskou, D. Radical scavenging activity of various extracts and fractions of sweet orange peel (Citrus sinensis). Food Chem. 2006, 94, 19-25. [CrossRef]

5. Adnan, M.; Azad, M.O.K.; Ju, H.S.; Son, J.M.; Park, C.H.; Shin, M.H.; Alle, M.; Cho, D.H. Development of biopolymer-mediated nanocomposites using hot-melt extrusion to enhance the bio-accessibility and antioxidant capacity of kenaf seed flour. Appl. Nanosci. 2019, 2019, 1-13. [CrossRef]

6. Bergman, M.; Varshavsky, L.; Gottlieb, H.E.; Grossman, S. The antioxidant activity of aqueous spinach extract: Chemical identification of active fractions. Phytochemistry 2001, 58, 143-152. [CrossRef]

7. Sahreen, S.; Khan, M.R.; Khan, R.A. Evaluation of antioxidant activities of various solvent extracts of Carissa opaca fruits. Food Chem. 2010, 122, 1205-1211. [CrossRef]

8. Zhao, S.; Li, X.; Cho, D.H.; Arasu, M.V.; Al-Dhabi, N.A.; Park, S.U. Accumulation of kaempferitrin and expression of phenyl-propanoid biosynthetic genes in kenaf (Hibiscus cannabinus). Molecules 2014, 19, 16987-16997. [CrossRef]

9. Kubmarawa, D.; Andenyang, I.F.H.; Magomya, A.M. Proximate composition and amino acid profile of two non-conventional leafy vegetables (Hibiscus cannabinus and Haematostaphis barteri). Afr. J. Food Sci. 2009, 3, 233-236.

10. Monti, A.; Alexopoulou, E. Kenaf: A Multi-Purpose Crop for Several Industrial Applications; Springer: Berlin/Heidelberg, Germany, 2013; ISBN 1447150678.

11. Jin, C.W.; Ghimeray, A.K.; Wang, L.; Xu, M.L.; Piao, J.P.; Cho, D.H. Far infrared assisted kenaf leaf tea preparation and its effect on phenolic compounds, antioxidant and ACE inhibitory activity. J. Med. Plant Res. 2013, 7, 1121-1128.

12. Ryu, J.; Kwon, S.-J.; Ahn, J.-W.; Jo, Y.D.; Kim, S.H.; Jeong, S.W.; Lee, M.K.; Kim, J.-B.; Kang, S.-Y. Phytochemicals and antioxidant activity in the kenaf plant (Hibiscus cannabinus L.). J. Plant Biotechnol. 2017, 44, 191-202. [CrossRef]

13. Son, J.M.; Ju, H.S.; Gung, H.J.N.; Azad, M.O.K.; Adnan, M.D.; Cho, D.H. Comparison of antioxidant active and useful components of the leaves extract fractions from Israeli Kenag (Hibisus cannabinus L.). J. Korean Soc. Med. Crop Sci. 2019, 27, 89.

14. Ryu, J.; Kwon, S.-J.; Ahn, J.-W.; Ha, B.-K.; Jeong, S.W.; Im, S.B.; Kim, J.-B.; Kim, S.H.; Lee, Y.-K.; Kang, S.-Y. Evaluation of nutritive value and identification of fungi in silage from new Kenaf (Hibiscus cannabinus) cultivars. Int. J. Agric. Biol. 2016, 18. [CrossRef] 
15. Adnan, M.; Azad, M.O.K.; Madhusudhan, A.; Saravanakumar, K.; Hu, X.; Wang, M.-H.; Ha, C.D. Simple and cleaner system of silver nanoparticle synthesis using kenaf seed and revealing its anticancer and antimicrobial potential. Nanotechnology 2020, 31, 265101. [CrossRef] [PubMed]

16. Harborne, A.J. Phytochemical Methods a Guide to Modern Techniques of Plant Analysis; Springer Science \& Business Media: Berlin, Germany, 1998; ISBN 0412572702.

17. Azad, M.O.K.; Park, B.S.; Adnan, M.; Germ, M.; Kreft, I.; Woo, S.H.; Park, C.H. Silicon biostimulant enhances the growth characteristics and fortifies the bioactive compounds in common and Tartary buckwheat plant. J. Crop Sci. Biotechnol. 2020, 2020, 1-9. [CrossRef]

18. Kalam Azad, M.O.; Jeong, D.I.; Adnan, M.; Salitxay, T.; Heo, J.W.; Naznin, M.T.; Lim, J.D.; Cho, D.H.; Park, B.J.; Park, C.H. Effect of different processing methods on the accumulation of the phenolic compounds and antioxidant profile of broomcorn millet (Panicum miliaceum L.) Flour. Foods 2019, 8, 230. [CrossRef]

19. Braca, A.; De Tommasi, N.; Di Bari, L.; Pizza, C.; Politi, M.; Morelli, I. Antioxidant principles from bauhinia $\mathrm{t}$ arapotensis. J. Nat. Prod. 2001, 64, 892-895. [CrossRef]

20. Atanasov, A.G.; Waltenberger, B.; Pferschy-Wenzig, E.-M.; Linder, T.; Wawrosch, C.; Uhrin, P.; Temml, V.; Wang, L.; Schwaiger, S.; Heiss, E.H. Discovery and resupply of pharmacologically active plant-derived natural products: A review. Biotechnol. Adv. 2015, 33, 1582-1614. [CrossRef]

21. Heffels, P.; Weber, F.; Schieber, A. Influence of accelerated solvent extraction and ultrasound-assisted extraction on the anthocyanin profile of different Vaccinium species in the context of statistical models for authentication. J. Agric. Food Chem. 2015, 63, 7532-7538. [CrossRef]

22. Adnan, M.; Chy, M.N.U.; Rudra, S.; Tahamina, A.; Das, R.; Tanim, M.A.H.; Siddique, T.I.; Hoque, A.; Tasnim, S.M.; Paul, A.; et al. Evaluation of Bonamia semidigyna (Roxb.) for antioxidant, antibacterial, anthelmintic and cytotoxic properties with the involvement of polyphenols. Orient. Pharm. Exp. Med. 2019, 19, 187-199. [CrossRef]

23. Pin, K.Y.; Chuah, A.L.; Rashih, A.A.; Mazura, M.P.; Fadzureena, J.; Vimala, S.; Rasadah, M.A. Antioxidant and anti-inflammatory activities of extracts of betel leaves (Piper betle) from solvents with different polarities. J. Trop. For. Sci. 2010, 22, 448-455.

24. Sim, Y.Y.; Ong, W.T.J.; Nyam, K.L. Effect of various solvents on the pulsed ultrasonic assisted extraction of phenolic compounds from Hibiscus cannabinus L. leaves. Ind. Crops Prod. 2019, 140, 111708. [CrossRef]

25. Yusri, N.M.; Chan, K.W.; Iqbal, S.; Ismail, M. Phenolic content and antioxidant activity of Hibiscus cannabinus L. seed extracts after sequential solvent extraction. Molecules 2012, 17, 12612-12621. [CrossRef] [PubMed]

26. Chatha, S.A.S.; Anwar, F.; Manzoor, M. Evaluation of the antioxidant activity of rice bran extracts using different antioxidant assays. Grasas Aceites 2006, 57, 328-335.

27. Sultana, B.; Anwar, F.; Ashraf, M. Effect of extraction solvent/technique on the antioxidant activity of selected medicinal plant extracts. Molecules 2009, 14, 2167-2180. [CrossRef]

28. Yi, T.; Li, S.-M.; Fan, J.-Y.; Fan, L.-L.; Zhang, Z.-F.; Luo, P.; Zhang, X.-J.; Wang, J.-G.; Zhu, L.; Zhao, Z.-Z. Comparative analysis of EPA and DHA in fish oil nutritional capsules by GC-MS. Lipids Health Dis. 2014, 13, 190. [CrossRef]

29. Huertas-Pérez, J.F.; Ernest, M.; Badoud, F. Quantification of folpet and phthalimide in tea and herbal infusions by LC-high-resolution MS and GC-MS/MS. Food Addit. Contam. Part A 2019, 36, 109-119. [CrossRef]

30. Search List. Available online: https://phytochem.nal.usda.gov/phytochem/search/list (accessed on 3 September 2020).

31. Do, Q.D.; Angkawijaya, A.E.; Tran-Nguyen, P.L.; Huynh, L.H.; Soetaredjo, F.E.; Ismadji, S.; Ju, Y.-H. Effect of extraction solvent on total phenol content, total flavonoid content, and antioxidant activity of Limnophila aromatica. J. Food Drug Anal. 2014, 22, 296-302. [CrossRef]

32. Abarca-Vargas, R.; Pena Malacara, C.F.; Petricevich, V.L. Characterization of chemical compounds with antioxidant and cytotoxic activities in bougainvillea $\mathrm{x}$ buttiana holttum and standl, (Var. rose) extracts. Antioxidants 2016, 5, 45. [CrossRef]

33. Abu, F.; Taib, M.; Norma, C.; Moklas, M.; Aris, M.; Mohd Akhir, S. Antioxidant properties of crude extract, partition extract, and fermented medium of dendrobium sabin flower. Evid.-Based Complement. Altern. Med. 2017, 2017. [CrossRef]

34. Boeing, J.S.; Barizão, É.O.; e Silva, B.C.; Montanher, P.F.; de Cinque Almeida, V.; Visentainer, J.V. Evaluation of solvent effect on the extraction of phenolic compounds and antioxidant capacities from the berries: Application of principal component analysis. Chem. Cent. J. 2014, 8, 48. [CrossRef] [PubMed] 
35. Samarakoon, K.W.; Lakmal, H.H.C.; Kim, S.Y.; Jeon, Y.-J. Electron spin resonance spectroscopic measurement of antioxidant activity of organic solvent extracts derived from the methanolic extracts of Sri Lankan thebu leaves (Costus speciosus). J. Natl. Sci. Found. Sri Lanka 2014, 42, 209-216. [CrossRef]

36. Adnan, M.; Chy, M.N.U.; Kamal, A.T.M.M.; Chowdhury, K.A.A.; Rahman, M.A.; Reza, A.S.M.A.; Moniruzzaman, M.; Rony, S.R.; Nasrin, M.S.; Azad, M.O.K.; et al. Intervention in neuropsychiatric disorders by suppressing inflammatory and oxidative stress signal and exploration of in silico studies for potential lead compounds from Holigarna caustica (Dennst.) Oken leaves. Biomolecules 2020, 10, 561. [CrossRef]

37. Danihelová, M.; Veverka, M.; Šturdík, E.; Jantová, S. Antioxidant action and cytotoxicity on HeLa and NIH-3T3 cells of new quercetin derivatives. Interdiscip. Toxicol. 2013, 6, 209-216. [CrossRef] [PubMed]

38. Guyton, J.R.; Lenz, M.L.; Mathews, B.; Hughes, H.; Karsan, D.; Selinger, E.; Smith, C. V Toxicity of oxidized low density lipoproteins for vascular smooth muscle cells and partial protection by antioxidants. Atherosclerosis 1995, 118, 237-249. [CrossRef]

39. Wong, Y.H.; Tan, W.Y.; Tan, C.P.; Long, K.; Nyam, K.L. Cytotoxic activity of kenaf (Hibiscus cannabinus L.) seed extract and oil against human cancer cell lines. Asian Pac. J. Trop. Biomed. 2014, 4, S510-S515. [CrossRef]

40. Chen, V.; Staub, R.E.; Baggett, S.; Chimmani, R.; Tagliaferri, M.; Cohen, I.; Shtivelman, E. Identification and analysis of the active phytochemicals from the anti-cancer botanical extract Bezielle. PLoS ONE 2012, 7, e30107. [CrossRef]

41. Khan, T.; Ali, M.; Khan, A.; Nisar, P.; Jan, S.A.; Afridi, S.; Shinwari, Z.K. Anticancer Plants: A review of the active phytochemicals, applications in animal models, and regulatory aspects. Biomolecules 2020, 10, 47. [CrossRef]

42. Vaquero, M.J.R.; Serravalle, L.R.T.; De Nadra, M.C.M.; De Saad, A.M.S. Antioxidant capacity and antibacterial activity of phenolic compounds from argentinean herbs infusions. Food Control. 2010, 21, 779-785. [CrossRef]

43. Kabir, M.I.; Adnan, M.; Rahman, M.M. Natural sources of tocotrienols: A note on absorption. J. Silico Vitr. Pharmacol. 2017, 3, 1-5. [CrossRef]

44. Adnan, M.; Chy, N.U.; Mostafa Kamal, A.T.M.; Azad, M.O.K.; Paul, A.; Uddin, S.B.; Barlow, J.W.; Faruque, M.O.; Park, C.H.; Cho, D.H. Investigation of the biological activities and characterization of bioactive constituents of Ophiorrhiza rugosa var. prostrata (D. Don) \& Mondal leaves through in vivo, in vitro, and in silico approaches. Molecules 2019, 24, 1367.

45. Patra, J.K.; Baek, K.-H. Antibacterial activity and action mechanism of the essential oil from Enteromorpha linza L. against foodborne pathogenic bacteria. Molecules 2016, 21, 388. [CrossRef] [PubMed]

46. Khan, R.; Islam, B.; Akram, M.; Shakil, S.; Ahmad, A.A.; Ali, S.M.; Siddiqui, M.; Khan, A.U. Antimicrobial activity of five herbal extracts against multi drug resistant (MDR) strains of bacteria and fungus of clinical origin. Molecules 2009, 14, 586-597. [CrossRef] [PubMed] 